\title{
Die Vorteile des Sprachverfalls. Zur Sprache der Lyrik im 19. Jahrhundert
}

I.

Sprachverfall ist an sich nicht zu messen. Er tritt im allgemeinen nur dann als solcher in Erscheinung, wenn er kontrastiv beleuchtet wird; denn er setzt Konventionen voraus, die nicht mehr eingehalten werden können, und das Bewußtsein dessen, daß dem so ist. Dabei wird die Differenz zwischen früher einmal gesetzter und jetzt nicht mehr erfüllbarer Norm in der Regel als Verlust bewertet, genauer: als solcher bewertet aus der Einsicht in das sprachliche Unvermögen der eigenen Zeit.

Betrachtet man Sprachverfall so, dann erlaubt das auch Überlegungen zu seinem zeitlichen Auftreten im Bereich des 19. Jahrhunderts. Es ist die Wende vom 18. zum 19. Jahrhundert, die den Boden bereitet für das Bewußtsein vom "Sprachverfall". Es kam auf, als die Idee des Niedergangs überhaupt aufgekommen war. Das hat seine eindeutigen historischen Gründe, und diese liegen nicht nur in der (nicht neuen) Erkenntnis der Gültigkeit biologischer Vorgänge auch im Bereich der Kultur. Die Idee vom Niedergang der eigenen Zeit ist zugleich Resultat von Geschichtserfahrungen, wie sie die Französische Revolution als Komplementärerscheinung zu allen Fortschrittsphantasien mit sich gebracht hatte. Zivilisationsermüdung war aber auch Regressionsphänomen einer gegen Ende des 18. Jahrhunderts fragwürdig gewordenen Aufklärung. Edward Gibbon schrieb, am römischen Schicksal das eigene exemplifizierend, das erste Standardwerk dieses Niedergangsbewußtseins mit seiner "History of the Decline and Fall of the Roman Empire" (1776-1788). Sie war in Deutschland gut bekannt. Über weite Strecken des 18. Jahrhunderts gab es kein Bewußtsein eines Sprachverfalls, weil es Verfallsbewußtsein an sich nicht gab. Im 19. Jahrhundert aber ist die These vom Kulturverfall der eigenen Zeit allgegenwärtig. Sprachverfall ist eines seiner Kennzeichen.

\section{1.}

Das Bewußtsein vom Sprachverfall zu Beginn des 19. Jahrhunderts ist also an besondere Voraussetzungen gebunden. Denn nicht jedes Ausufern und nicht jede Erstarrung müssen Sprachverfall sein: Gehören Formalismus oder Schwulststil wie im 17. Jahrhundert zum Normensystem einer Sprache, sind die genannten Phänomene also intentionalisiert oder konventionalisiert, so sind sie nicht Niedergangserscheinungen. Auch eine 
mehr oder weniger weitverbreitete Sprachkritik ist noch kein Zeichen von Sprachverfall. Das Gegenteil ist richtig. Denn in Zeiten funktionierender Normensysteme gibt es zwar immer wieder Abweichler, aber dieses Abweichlertum wird, sofern es überhaupt zur Kenntnis genommen wird, in der Regel sofort von den Positionen eines intakten Normensystems aus kritisch begutachet: Einem (möglichen) Verfall wird also unverzüglich Einhalt geboten, und wer die Rolle der Kritik etwa im 18. Jahrhundert kennt, der weiß, daß damit jedes Ausscheren aus der Norm mit der ganzen Macht philosophischer Urteilssprüche unterdrückt wurde. Sprachkritik, wie sie im 18. Jahrhundert geübt wird, ist immer auch ein Appell an eine für überall und immer durchsetzbar gehaltene Sprachkultur bei nicht bezweifeltem Wissen um die Möglichkeit wie auch um die Notwendigkeit einer solchen.

Ein Beispiel einer noch funktionierenden Sprachkritik und eines noch intakten Normenbewußtseins bietet Schillers Kritik an Bürgers Gedichten von 1791. Schiller konstatiert zwar schon ausdrücklich „den Verfall der lyrischen Dichtkunst" - und damit den der lyrischen Sprache. ${ }^{1}$ Aber er antwortet auf diesen Verfall mit einem lyrischen Kulturprogramm, das er wie ein Bollwerk dagegen aufrichtet. Mit anderen Worten: Der lyrische Dichter erfährt von ihm genau, was er zu sein und zu schreiben hat, will er als solcher vor der literarischen Kritik bestehen. Der wichtigste Eckpfeiler dieses Bollwerks lautet "Veredelung”, und unter Veredelung versteht Schiller (in der pathetischen Sprache seiner Zeit) einen LäuterungsprozeB „zur reinsten herrlichsten Menschheit”, dem die Individualität zu unterwerfen ist. Diese muß generisch werden, das Subjektive zum „ächt Objektiven", alles Vereinzelte und Ich-Bezogene also ausgelöscht, das Genotypische hingegen hervorgekehrt werden. Wer das nicht weiß, kann weder mit Schillers Balladen noch mit seinen philosophischen Gedichten etwas anfangen. Bürger nun, der sogenannte Volksdichter, weicht davon ab, kann nicht „das Individuelle und Lokale zum Allgemeinen" erheben, und so zieht er sich Schillers Kritik zu. Schiller verdammt im gleichen Atemzug auch noch den Leser, der an Liedern Gefallen findet, "worin noch der ganze trübe Strudel einer ungebändigten Leidenschaft braust und wallt und mit dem Affekt des begeisterten Dichters auch alle seine eigentümlichen Geistesflecken sich abspiegeln".2 Darin mag vielleicht ein gutes Stück Schillerscher Selbstkritik verborgen sein, auf die eigene vormalige Sturm und Drang-Dichtung bezogen. Aber Schiller urteilt, was Bürger angeht, nicht nur aus dem Bewußtsein einer erfolgrei-

1 Schiller 1958, S. 245.

2 Schiller 1958, S. 261. 
chen Selbstüberwindung, sondern hat auch einen Trumpf, den er gegen den Kritisierten ebenso rücksichtslos wie verständnislos ausspielt. Denn er verfügt über Grundsätze und erinnert den Kontrahenten daran, daß der diese Grundsätze „im Ernst nicht wohl leugnen, nicht miBverstehen” könne, "ohne seine Begriffe von der Kunst verdächtig zu machen". ${ }^{3}$ Hier haben wir das Normensystem in seiner Rigorosität, und mit ihm zieht Schiller zu Felde gegen einen lyrischen Anarchisten, einen poetischen Nonkonformisten. Vom Sprachverfall wird man angesichts eines so intakten Systems und so unbezweifelter Grundsätze nicht sprechen können. Der Abweichler wird unmittelbar kritisch in die vorhandene Sprachnorm integriert. Auch ein Verdammungsurteil wie das Schillers ist noch ein Integrationsversuch - freilich mit negativen Vorzeichen.

Aber kommt Verfall nicht schon an den Rändern dieses Sprachimperiums hoch? Der Sprachnorm hatte sich jeder zu fügen. Doch sie war nicht länger Maßstab für Dichter von Profession. Die Aufklärung hatte den Geltungsbereich ihrer Normen, am Zeitalter des Barock und der galanten Poesie gemessen, rigoros erweitert und ihnen eine quasi kulturelle Grundgesetzlichkeit zuerkannt. In Verbindung mit dem unbeschränkten Zutrauen der Aufklärung in die unbeschränkte Ausdrucksmöglichkeit des Menschen aber bedeutete das, daB dem Egalitätsanspruch des Zeitalters zufolge im Prinzip jeder das leisten sollte, was nicht jeder leisten konnte. Schiller schrieb noch 1799: ,Jeder gebildete Mensch muB seine Empfindungen poetisch schön ausdrücken und folglich ein gutes Gedicht (lyrisches) machen können". 4 Aber das war bereits der Anfang vom Ende. Zumindest wurde hier die Tür für den Sprachverfall aufgestoßen. Denn im Gefolge dieser Gutgläubigkeit, was die Erziehungsfähigkeit des Menschen anging, erschien der Typ des Dilettanten, stellte sich das Problem der zwar notwendigen, aber nicht mehr möglichen Einhaltbarkeit der poetischen Norm - und damit das des Normenverfalls, das zum Sprachverfall führen mußte. Dilettantismus ist eine der direktesten Begleiterscheinungen der Aufklärung, weil er die Umsetzung jener egalitär-erzieherischen Vorstellung ist, die Schiller als allgültige Maxime formuliert hatte. Gegen Ende des 18. Jahrhunderts sollte ihr jeder, konnte ihr aber nicht jeder genügen. Norm und Normerfüllung wichen bedrohlich oft voneinander ab.

Das hätte zwar noch nicht unbedingt zu Sprachverfall führen müssen. Doch es kam noch etwas hinzu, was ihn vielleicht stärker noch als alles

3 Schiller 1958, S. 262.

4 Schiller 1963, Anhang: Schemata über den Dilettantismus (7). Lyrische Poesie. 
andere beschleunigte: das auffällige Fehlen nachmeßbarer, verständlicher und damit bewußt befolgbarer Maßstäbe gegen Ende des 18. Jahrhunderts. Zwar gab es schon lange keine Poetiken mehr, keine lyrischen Gesetzbücher, die Regeln boten und Anwendungsmodalitäten lehrten. Es gab nur eine Macht, die angesichts der aufklärerischen Ausweitung der lyrischen Produktivität dem Wildwuchs, der damit zwangsläufig drohte, Einhalt gebieten und wenn nicht Normen, so doch Muster liefern sollte: die der Tradition. Schiller hat das ebenso klar wie kompromißlos formuliert, als er schrieb: „Da es nun keine objective Gesetze weder für das innere noch für das äusere eines Gedichts noch giebt, so müssen sich die Liebhaber strenger noch als die Meister an anerkannte gute Muster halten und eher das Gute was schon da ist nachahmen als nach Originalität streben". ${ }^{5}$ Die Tradition sollte also im ausgehenden 18 . Jahrhundert das ersetzen, was Regelbücher im 17. Jahrhundert waren, und Tradition war auch, wie Schiller wußte, die "einer reichen cultivierten Dichtersprache". Doch es zeigt sich, daß im Zeitalter des Dilettantismus die Orientierung an Mustern allein offenbar nicht mehr ausreichte, und hier wird etwas von den spezifischen Bedingungen des Sprachverfalls am Ausgang des 18. Jahrhunderts sichtbar. Denn gerade die Traditionsbezogenheit, die eigentlich dem Sprachverfall entgegenwirken sollte, stellte sich als eine seiner wesentlichen Ursachen heraus. Der Reichtum der kultivierten Dichtersprache machte sie für alle verfügbar, und so wurde sie zum großen Selbstbedienungsladen, dem jeder entnehmen konnte, was er brauchte - die Sprachfülle erschien als Uberfülle, Tradition als Zusammenflub verschiedenartigster Traditionen, und der anerkannten guten Muster gab es offenbar zu viele, als dab eines oder einige selektiv für vorbildlich erachtet werden konnten. Poesieklitterung war das Ergebnis, und Schiller sah sich der Erkenntnis konfrontiert, daß sein Heilmittel ein gefährliches Gift war. Die Orientierung an möglichst allen guten Mustern bedeutete Beliebigkeit; sie führte direkt in einen ebenso weitverbreiteten wie fragwürdigen Alexandrinismus.

Schiller hat den damals schon drohenden Sprachverfall durch das Überhandnehmen der Verfügbarkeit und der Beliebigkeit in der Aneignung von Traditionen sehr wohl gesehen. Er notierte - und seine Darstellung ist nichts anderes als die Beschreibung eines Sprachverfalls -: ${ }_{n}$ Alle Dilettanten sind Plagiarii. Sie entnerven und vernichten jedes original schöne in der Sprache und im Gedanken, indem sie es nachsprechen, nachäffen und ihre Leerheit damit ausflicken. So wird die Sprache nach und nach mit zusammen geplünderten Phrasen und Formeln angefüllt, die nichts mehr sagen, und man kann ganze Bücher lesen, die schön

5 Schiller 1963, Anhang. 
stilisiert sind und gar nichts enthalten. Kurz alles wahrhaft schöne und gute der ächten Poesie wird durch den überhandnehmenden Dilettantism profaniert, herumgeschleppt und entwürdigt". ${ }^{6}$ Wie schwierig Schillers Bastion zu halten war, zeigt sich am Begriff des „original schönen”. Er ist nicht definiert, und er kann gar nicht definiert werden. Wer darüber entscheidet, ist nicht ein poetisches Gesetz oder eine Vorschrift, sondern neben den Mustern, die aber an sich nicht mehr viel besagen, der Kritiker, der in dieser Zeit gewissermaßen noch als philosophische Inkarnation der poetischen Normen gilt: der Kritiker als Richter und Regelinterpret. Hier tritt die ausführende Instanz - ein Verfallsphänomen eigener Art an die Stelle des Gesetzes, die Exekutive spricht dort, wo die Legislative sprechen müBte, weil die Gesetze selbst nicht mehr definierbar sind. „Unwissende Selbstherrlichkeit einer poetisch mangelhaften Diktion" ist Kennzeichen dieses Dilettantismus, Sprachverfall seine Begleiterscheinung. „Schöngeisterey”, „Belletristerey", „Frauenzimmergedichte”, "belletristische Flachheit und Leerheit", „Nullität", Geistlosigkeit und Gehaltlosigkeit, "unruhiger Productionsbetriebe" - das sind die Injurien, die Schiller dem Dilettantismus nachruft, und es ist zugleich das Urteil einer poetischen Zensur, die freilich mit dem Ende des Jahrhunderts auch an das Ende ihrer Gültigkeit gekommen ist. Andere Charakteristika sind „Flachheit", „Gedankenleerheit”, „Sinnlichkeit”, "Karikatur", "falsche Kennerschaft", „Mittelmäßigkeit”, ,Verderbnis des Geschmacks", "Vorliebnehmen mit dem Schein”, „Pfuscherey”, letzteres vor allem dort, wo eine ausgebildete Sprache fehit. Das ist der Fall in Deutschland, und Schillers Dichterkritik schlägt geradezu in Nationalkritik um, wenn er schreibt: „Daß die deutsche Sprache durch kein großes Dichtergenie sondern durch bloße mittelmäßige Köpfe anfieng zur Dichtersprache gebraucht zu werden, muBte dem Dilettantism Muth machen, sich gleichfalls darinn zu versuchten". ${ }^{7}$

Aus dem Phänomen des Dilettantismus heraus sind also die wichtigsten Bedingungen für den Sprachverfall in der ersten Hälfte des 19. Jahrhunderts zu benennen: zum einen Traditionsverlust in der Sonderform eines Übermaßes an Traditionen, das Unverbindlichkeit bedeutet, d.h. ein gleichsam „leeres" Traditionsbewußtsein ohne Geschichtsbindung, das zwangsläufig zu lyrischer Klitterei führen mußte; sodann das Ende einer literarischen Kritik, die sich zwar noch auf ihren hohen Rang beruft, ohne sich jedoch überzeugend begründen zu können; drittens aufkommender Subjektivismus als Gegenreaktion auf die Ära des „ächt Objek-

\footnotetext{
6 Schiller 1963, Anhang.

7 Schiller 1963, Anhang.
} 
tiven". Das sind Komplementärerscheinungen des Zusammenbruchs des alten Normensystems, wie sich das etwa auch an der ausufernden Gattungsvielfalt, am Aufkommen neuer literarischer $\mathrm{Z}$ weckformen, an der vollkommenen Diffusion der Begriffe im Bereich der Poetik abzeichnet (Roman und Novelle sind nicht mehr voneinander unterscheidbar, poetische Definitionen, so meinte Karl Gutzkow, „wie auf der Flucht"). ${ }^{8}$

Zeitgenössische Äußerungen dazu finden sich wiederholt. So hat Heine die satirische Geißel über die schwäbische Dichterschule geschwungen, die nur noch „das schöne Wetter besinge, die Frühlingssonne, die Mayenwonne, die Gelbveiglein, und die Quetschenbäume". Das war literarische Kritik am Sprach- und Imaginationsverfall einer schwäbischen Provinziallyrik, und wir wissen, wie sehr Heine sie auch anderswo geübt hat: nicht zuletzt am Grafen Platen, an dem er das kritisierte, was der angesichts der überreichen literarischen Traditionen nur zu selbstverständlich nutzte. Daß jener kein Dichter sei, ist der ritournellartige Refrain in seiner Streitschrift gegen Platen, in der er den lyrischen Seiltänzer als poetischen Kompilator entlarvt: „Wenn der Graf Platen noch so hübsch in den Ghaselen seine schaukelnden Balancierkünste treibt, wenn er in seinen Oden noch so vortrefflich den Eiertanz exekutiert, ja, wenn er, in seinen Lustspielen, sich auf den Kopf stellt - so ist er doch kein Dichter". ${ }^{10}$ Platen tut das, was Schiller unbedingt dem Dilettantismus zugerechnet haben würde: Er vermischt hemmungslos Traditionen. So heißt es bei Heine: „Graf Platen hingegen, trotz seinem Pochen auf Klassizität, behandelt seinen Gegenstand vielmehr romantisch, verschleiernd, sehnsüchtig, pfäffisch, - ich muß hinzusetzen: heuchlerisch [...]". Platen will Iliaden und Odysseen erfinden und erweist sich gerade darin als Dilettant in der Erscheinungsform des 19. Jahrhunderts, also als Epigone - ein Alexandrinist reinsten Wassers, der in den lyrischen Traditionen herumrührt, bis er sein fragwürdiges Gebräu zusammengekocht hat. Das kann er nicht nur schneller als andere, sondern auch sehr viel schlechter. So bleibt er ein Poet, der „sich ghaselig hingibt in windiger Weichheit"11 - „in mühsam gefeilten Versen", wie sich versteht, ein höchst zweifelhafter poetischer Sudelkoch, eben "kein Dichter".

Lyrischer Verfall? Sprachverfall, Verfall an Ausdrucksfähigkeit, an Überzeugungskraft, an Individualität, an Substanz? Wir sind gewohnt, Lyrik

8 Gutzkow o.J., Bd. 8, S. 114.

${ }^{8}$ Heine 1978 , S. 154.

10 Heine o.J., S. 353.

11 Heine o.J., S. 355 u. 357. 
des 19. Jahrhunderts über relativ weite Partien hin so zu lesen, und an Beispielen fehlt es nicht. Die Auswahl fällt schwer, nicht weil es zu wenig, sondern weil es zu viel gibt. Einige Beispiele mögen das demonstrieren.
Wär' ich ein muntres Hirschlein schlank,
Wollt' ich im grünen Walde gehn,
Spazieren gehn bei Hörnerklang,
Nach meinem Liebsten mich umsehn.

Der Verfasser dieser Verse, hinter denen man wohl nicht allzuviel lyrischen Tiefsinn vermuten darf: Joseph von Eichendorff. ${ }^{12}$

Oh süß Kind, Geliebte, Schwester,
Schatten, Leben, Leid und Lust,
Alle Vöglein haben Nester,
Und mein Herz hat eine Brust.

Das ist eine Strophe aus dem Gedicht "Im Wetter auf der Heimfahrt", der Verfasser: Clemens Brentano. ${ }^{13}$ Dann ein Bundeslied:

Geist des Bundes, schwebe nieder,

Deines Altars Flammen glühn,

Aus den Augen meiner Brüder

Seh ich Opferflammen sprühn.

Hörst Du unsere Hymnen tönen?

Sie verkünden deinen Ruhm;

Komm herab zu deinen Söhnen

In Germanias Heiligtum.

Der Verfasser: Wilhelm Hauff. ${ }^{14}$ Oder:

Es blasen die blauen Husaren,

Und reiten zum Thor hinaus;

Da komm'ich, Geliebte, und bringe

Dir einen Rosenstrauß.

Der Verfasser: Heinrich Heine. ${ }^{15}$

Ihr Ritter, die ihr haus't in euren Forsten, ist euch der Helmbusch von dem Haupt gefallen?

Versteht ihr nicht den Panzer mehr zu schnallen?

Ist ganz die Rüstung eures Muth's zerborsten?

Der Verfasser: Friedrich Rückert. ${ }^{16}$

12 Eichendorff 1957, S. 207.

13 Brentano 1968, S. 572.

14 Hauff 1970, S. $363 f$.

15 Heine 1975, S. 289.

16 Rückert 1868, S. 9. 
Zum Schluß dieser poetischen Blütenlese noch ein ganzes Gedicht:

Du Rose, wenn du neidenswerth willst sterben,

So lass' frühmorgens pflücken dich vom Strauche,

Bevor sich an des Mittags Gluthenhauche

Die duftigen Schimmer deiner Wang' entfärben.

Und sinkt der Mittag müde auf die Matten, Rast' ich am Bächlein in den kühlsten Schatten, Ein leises Flüstern geht in allen Bäumen,

Das Bächlein plaudert wirre wie in Träumen,

Da saßen wir beisammen, still und traurig,

Und sahn uns an, und wurden immer traur'ger.

Die Eiche säuselte wie Sterbeseufzer,

Tiefschmerzlich sang die Nachtigall herab.

Nun schleichen aus dem Moore kühle Schauer

Und leise Nebel übers Heideland;

Der Himmel ließ, nachsinnend seiner Trauer,

Die Sonne lässig fallen aus der Hand.

Dieses Gedicht hat keinen Verfasser, oder vielmehr es hat ihrer vier. Sie heißen Rückert, ${ }^{17}$ Eichendorff, ${ }^{18}$ Heine $^{19}$ und Lenau. ${ }^{20}$

Diese Kompilation ist zweifellos lyrischer Frevel. Aber solch ungutes Tun wird dem Leser leichtgemacht. Und daß es so leichtgemacht wird, scheint ein Zeichen eben des Sprachverfalls zu sein. Ganze Strophen sind konvertibel, Gedichte achtbarster Lyriker lassen sich ineinanderschieben, und so könnten etwa aus zwanzig alten Naturgedichten ebensoviele neue entstehen, ohne daß irgendeine Zeile neu geschrieben worden wäre.

Sprachverfall? Vermutlich könnten auch anakreontische Gedichte ineinandergeschachtelt werden, und nicht weniger leicht ließen sich Zeilen von Gryphius-Gedichten solchen von Hofmannswaldau implantieren. Und doch würde es sich um sehr verschiedene Dinge handeln. Barocklyrik läßt das Formelwesen und damit Austauschbarkeit ausdrücklich zu. Individuelles ist (wie noch bei Schiller) verpönt, Generisches hochwillkommen. Und das Ordo-Denken sichert den Barockgedichten ein gemeinsames Bezugssystem, auf das sich Gryphius wie Lohenstein beziehen können.

17 Rückert 1868, S. 339.

18 Eichendorff 1957, S. 81.

19 Heine 1975 , S. 311

20 Lenau 1971, S. 64. 
In 19. Jahrhundert aber ist an die Stelle dieses einheitlichen Bezugssystems eine Vielfalt von beliebigen Kombinationsmöglichkeiten getreten. Auffälliger noch ist die Diskrepanz zwischen Aussage und Form, auch die relative Belanglosigkeit vieler Themen. Wo Zeitprobleme auftauchen, etwa bei Eichendorff oder Rückert, sind sie in einen lyrischen Bombast eingewickelt, der den eigentlichen Anlaß des Gedichtes oft völlig verkennen läßt. Es gelingt der Lyrik denn auch, zuweilen über etwas hinwegzutäuschen, worüber Prosa und Drama sehr viel weniger hinwegtäuschen: die innere Widersprüchlichkeit der Zeit. Die Zahl der Äußerungen über sie ist Legion, und sie ziehen sich quer durch alle poetischen Fraktionen und politischen Gruppierungen hindurch. Die Lyrik ist weitgehend davor bewahrt geblieben, sie stellt sich nicht selten als poetisches Himmelreich dar, in dem die Rechnungen alle noch aufgehen. Die literarische Revolution, die nahezu alle Gattungen in dieser Zeit erfaBt hat, hat um die Lyrik einen relativ großen Bogen gemacht. Heine ist die große Ausnahme, nicht die Regel, denn diese wird verkörpert von Platen und Vischer, Zimmermann und Lenau. „Jetzo ist die Zeit der Kleinen”, dichtete Immermann in seinem "Tulifäntchen". Man wird, was die Masse der lyrischen Gedichte in der ersten Hälfte des 19. Jahrhunderts angeht, dieser Bestandsaufnahme kaum widersprechen können.

\section{III.}

Haben wir richtig argumentiert? Wir haben es nur insoweit, als wir der weitverbreiteten, seit René Wellek populären These gefolgt sind, daß das Wesen einer literarischen Epoche durch ein Normensystem geprägt sei und dab dieses ihre Unverwechselbarkeit wie auch ihren Rang ausmache. $^{21}$ Mag eine Feststellung wie die Welleks zunächst auch nur identifikatorischen Charakter haben, so schleichen sich damit unvermeidlich aber auch wertende Vorstellungen ein, und eben sie gaben oft Anlab zur Abwertung des 19. Jahrhunderts. Sie ist auch heute noch weitverbreitet. Doch ist Wertabfall von einem Normensystem tatsächlich ein Untergangsphänomen? Sprachverfall kann auch Sprachbefreiung bedeuten. Und reagiert nicht die Sprache nach 1800 auf das Ende der klassischen Normen geradezu mit einem gleichsam verbalen Freudentaumel? Vieles spricht dafür, daß die vorangegangene Konvention plötzlich als verhabte Diktatur empfunden wurde, von der man sich befreit sah. Was vorher geordnet und wohlkanalisiert erschien, schwemmte plötzlich über die Ufer, machte sich in einer scheinbar anarchischen Sprachvielfalt deutlich. Wir sind beim zweiten Teil eines Themas, das in der Tat janusgesichtig ist wie kaum etwas anderes, was die Sprachgeschichte des 19. Jahrhunderts

21 Wellek/Warren 1956, S. 24. 
angeht: bei den Vorteilen des Sprachverfalls.

Mit der schönen objektiven Welt war es in der Tat zu Ende, aber was Schiller als Verlust beklagt hatte, haben Heine und die Romantiker mit Frohlocken begrüßt. Der beginnende Subjektivismus ist auch ein sprachlicher Subjektivismus, und Heine ist sein vermutlich wichtigster Fürsprecher. Ein Ich-Perspektivismus löst die allgemeinen Feststellungen ab, und das Ich, das sich sprachlich ausdrückt, ist nicht, wie in der Aufklärung, ein mehr oder weniger normiertes Ich mit generellen Fähigkeiten und Lernbedürfnissen, sondern eine unteilbare Einzigartigkeit. Das drückt sich sofort in einer Neigung zu Neologismen aus, die ja immer ein gleichzeitig beredter und stummer Protest gegen sprachliche Normensysteme sind. Heine, wohl einer der am stärksten sprachschöpferischen Lyriker des frühen 19. Jahrhunderts, läBt das eindrucksvoll in seinen "Nordsee”Zyklen erkennen. Das zweite Gedicht des Ersten Zyklus beginnt mit: Am blassen Meeresstrande / Saß ich gedankenbekümmert und einsam. ${ }^{22}$ Das sind noch nicht Neologismen im strengen Sinne, aber der Neologismus ist im frühen 19. Jahrhundert ohnehin ein vieigestaltiges Phänomen. Man wird auch Wendungen wie den blassen Meeresstrand ebenso dazurechnen wie die bei Heine zahlreich aufkommenden Synästhesien. Als er oder vielmehr das lyrische Ich nun derart am Meeresstrande sitzt, hört er - Lautmalereien seien hier übergangen - ein wiegenliedheimliches Singen. Das ist eine fesselnde Neuprägung, wenngleich wir zugeben müssen, daß sie ihre gedanklichen Unschärfen hat. Aber von dieser Art begegnet vieles in den "Nordsee"-Zyklen. Die Mädchen haben neugierkluge Augen $^{23}$ und sie hören zu mit kleinen, horchenden Herzen. Die Sonne neigt sich hinab in's weitaufschauernde, silbergraue Weltenmeer. Silbergrau ist die Luft oder vielmehr der Abendduft bei Dutzenden anderer Dichter auch, aber das weitaufschauernde Weltenmeer ${ }^{24}$ findet sich vorher nicht. Stolze, glückgehärtete Menschen, ${ }^{25}$ der Sonnengott und sein fluthenkaltes Wittwerbett, der Nordwind, der ungestaltet über dem Meer, platt auf dem Bauch liegt, ${ }^{26}$ die tollen Geschichten, die er erzählt, dunkeltrotzig und zaubergewaltig, der fiuthbefeuchtete Sand - eine sehr subjektiv gesehene und sprachlich subjektiv ausgedrückte Welt. Wenn vom weißen Tanz der Wellen die

22 Heine 1975, S. 359.

${ }^{23}$ Heine 1975, S. 361.

24 Heine 1975, S. 361.

25 Heine 1975, S. 363.

${ }^{26}$ Heine 1975, S. 365. 
Rede ist, ${ }^{27}$ von der wild ertọsenden Flut, dann wissen wir zwar, daß das wohl schon um 1820 verbrauchte Formulierungen waren. Davon ist damals kein Dichter frei, Heine weniger als andere. Aber wenn im Sturm die weitgähnenden Fluthabgründe erwähnt sind, ${ }^{28}$ wenn vom smaragdenen Frühling gesprochen ist ${ }^{2 \theta}$ und von der schwarzen Son$n e^{30}$ - fast ein expressionistisches Bild - , von den Mōwen als Lüfteseglern, ${ }^{31}$ von den Riesengestalten der Wolken im luftigen Pantheon, ${ }^{32}$ dann sind das so unerhört neue Wendungen, daß wir gerne die schimmernden Segel ${ }^{33}$ oder die weißgekräuselten Wellen in Kauf nehmen da zahlt Heine auch einmal mit kleiner abgegriffener poetischen Münze. Aber seine Neuprägungen sind beachtlich. Denn hier ist, stärker noch als etwa im Sturm und Drang, ein Protestpotential verbalisiert, das sich nicht, wie damals, gegen die allgemeine Feldzugslinie der Aufklärung richtete, sondern gegen die hochstilisierte Sprache der Klassik.

Man wäre schlecht beraten, wollte man dieses Abgleiten von der Norm als Sprachverfall klassifizieren; vielmehr erscheint gerade die klassische Norm als eine schon verfallene Sprache, und das gibt zu bedenken, ob Begriffe wie 'Sprachnorm' und 'Sprachverfall' im frühen 19. Jahrhundert in ihrem Bedeutungsgehalt nicht vielmehr völlig reziprok angewandt werden müssen. Um es überspitzt zu sagen: Die von Schiller noch so leidenschaftlich propagierte Sprachnorm ist im Grunde bereits Sprachverfall, der landläufige Sprachverfall hingegen etwas Lebendiges, Freiheit, Aufbruch oder, um es mit einer biologistischen Formel zu benennen, Evolution. Evolution aber ist immer antinormativ, Norm und Normensysteme hingegen sind Erstarrungen, signalisieren ein Zur-Ruhe-Kommen der Sprache, und es sieht so aus, als ob derartige Norm-Zustände dazu neigten, ihre unerbittlichen Anwälte und gesetzestreuen Ankläger zu finden, die alles verurteilen, was nicht Erstarrung ist. Neologismen sind in dieser Zeit im Unterschied zum Pietismus subjektbezogene Aussageversuche, mit nichts anderem im Hintergrund als dem eigenen Erlebnishorizont, oder sagen wir besser: dem eigenen Sprachvermögen, das eben

\footnotetext{
27 Heine 1975, S. 373.

Heine 1975, S. 379.

29 Heine 1975, S. 397.

30 Heine 1975, S. 403.

31 Heine 1975, S. 407.

32 Heine 1975, S. 413.

33 Heine 1975, S. 421.
} 
nicht mehr einer Norm angepaßt wird, sondern der Realität, und wenn es tatsächlich nach dem angeblichen Verfall der Sprachnorm der Klassik so etwas wie eine Sprachkrise gegeben haben sollte, so ist es eine produktive Krise gewesen mit dem Ziel, der subjektiv erlebten Wirklichkeit sprachlich so nahe wie möglich zu kommen, wie das eben Heine in seinen Nordsee-Zyklen so einleuchtend demonstriert. Wir haben ähnliche Beispiele bei der Droste und bei Mörike.

\section{IV.}

Literarische und sprachliche Umbruchperioden - und das 19. Jahrhundert ist eine solche gewesen - führen nicht selten dazu, daß konträre Tendenzen freigesetzt werden. Normative Systeme und Wertvorstellungen haben immer nur eine sehr begrenzte Bandbreite, denn es liegt in der Natur der Sache, daß Normabweichungen, Variationen oder auch Erweiterungen nichts Neues zulassen und eben dieses als das definieren, was sie aus der Sicht der Normsetzung sind: als Sprachverfall. Im 18. Jahrhundert aber, nach dem Ende der Normen, lassen sich zum wachsenden Realitätsbezug in der Sprache auch gegenläufige Tendenzen von ungefăhr gleicher Stärke ausmachen, und gelegentlich finden diese sich sogar in den Werken ein- und desselben Dichters. Um es vereinfacht zu sagen: Den Annäherungen an die Realität entspricht eine Abwendung von der Realität, freilich nicht ins Ideale oder objektiv Schöne hin, sondern in das Spiel mit anderen Sprachen, literarischen Formen, Kulturen. Bei allem Interesse Schillers am Spiel und der Freiheit des Spiels: Normen lassen ein solches im Grunde genommen nicht zu. Normen sind auch Sprachfesseln, und in der Lyrik des 19. Jahrhunderts findet sich der verspätete, aber um so nachdrücklichere Protest dagegen. Eine poetische Entgrenzung findet statt, und sie macht an den Grenzen Europas durchaus nicht halt. Das Wiederaufkommen des Petrarkismus ist kein Zufall: Heine und Mörike, Storm und Rückert sind gleichermaßen zumindest in Teilen ihrer Lyrik Petrarkisten.

Ein besonders gutes Beispiel bietet die Lyrik von Rückert. Rückert hat diverse poetische Sträuße zusammengebunden; einer ist "Liebesfrühling" betitelt, dazu kommen noch die Agnes-Lieder und die Amaryllis-Lieder. Der „Liebesfrühling” ist, vom Standpunkt eines wertüberzeugten Lyrikbegriffes her gesehen, reinster Kitsch. Die Stadien des Rückertschen Liebesfrühlings würden jedem Lore-Roman Ehre machen. Sie reichen von "Erwacht" und „Geschieden" über "Gemieden”, „Entfremdet” bis hin zum "Wiedergewonnen" und "Verbunden". Das ist eine ebenso lange wie unglaubwürdige lyrische Affäre, dutzendfach variiert in alle nur möglichen und denkbaren Verästelungen hinein, und selbst in juveni- 
ler Verblendung würde man nicht auf die Idee kommen, man habe es hier mit persönlichen Bekenntnisgedichten zu tun. Dazu ist alles viel zu leichtfüBig. Liebste und Liebchen sind Dutzendworte, und überall stehen die Linden zu dicht, ist in zu vielen Gedichten ständiger Frühling, spricht die Geliebte allzu leichtzüngig bei des Mondes stillem Leuchten, und die Rosen, altes Liebessymbol, haben hier nur eine Funktion: Sie sollen die Liebesgluten, die völlig unglaubhaften, farbkräftig verdeutlichen. Rückert ist mit unendlicher Variationslust ausgestattet, und er tut es, auf allerdings recht säkularisierte Weise, den alten Pietisten nach, wenn er ein Gedicht mit den beiden Zeilen beginnen läft: Ich seh's an allen Zeichen, / Daß meine Sonne kommt. ${ }^{34}$ Sie kommt, sie naht, erwacht, erblüht, erscheint; sie ist sein, sein allein, gehört nur ihm. Das Ende ist ein lyrisch-erotischer Sonnenbrand, und der Leser fragt sich, warum so viele lyrische Umwege nötig gewesen sind, um die Geliebte als Sonne zu beschreiben. Aber gerade die Schwäche dieses Gedichtes ist zugleich sein eigentlicher Gewinn: Denn auch der lyrische Tölpel merkt sehr bald, daß es hier gar nicht um die Geliebte geht, sondern daB hier die Variationskunst und Variationslust die treibenden poetischen Motoren sind, und läßt man sich von denen mitbewegen, hat man am Ende sogar sein Vergnügen an der lyrischen Drechselkunst.

Doch Rückerts lyrische Sprache ist alles andere als einfallslose Reimerei. Sucht man nach Wirklichkeitsbezügen, tappt man zwar im Leeren herum. Die Welt ist völlig entsubstantialisiert, und dazu paßt, daß im gleichen Zyklus nicht von einer, sondern gleich von tausend Nachtigallen die Rede ist, von tausend Frühlingsrosen, tausend Liebessternen und tausend Edelsteinen. Eine Inflation, ohne Zweifel, aber wir haben es hier nicht mit einem Ausverkauf an eigenen Empfindungen zu tun. Wir haben es vielmehr mit lyrischen Schreibübungen zu tun, sie sollen die Sprache gelenkig erhalten. Stoßen wir auf Formeln wie Schiffer der Liebe, so mögen wir uns fragen, ob das eine kühne oder eine verunglückte Metapher ist, ein niedliches lyrisches Spiel oder ein kleiner lyrischer Betriebsunfall. Aber wir werden der Entscheidung enthoben durch die Einsicht, daß hier die sprachliche Verfügbarkeit das lyrische $\mathrm{MaB}$ aller Dinge ist. Was Rückert recht ist, ist Heine in seinem "Buch der Lieder" nur billig. Vielleicht sind es nicht tausend Frühlingsrosen, aber die Rose und das rosenfarbige Gesicht der Geliebten, die blauen Äuglein und der schneeweiße $\mathrm{Hals}$ begegnen auch dort.

Wirklichkeitsbezug gibt es in diesem Teil von Heines „Buch der Lieder" nicht, und ebensowenig ist glaubhaft, was Rückert schreibt. Wenn

34 Rückert 1868, S. 464. 
der uns lyrisch versichert, daß er am Bach wetteifernde Hirtengesänge gehört habe, daB sich seine Brust schwellend gehoben habe, als er von Liebesleid und Sommerlust erfuhr, wenn er von der verklärten Lichtnatur spricht, vom tönenden Schmuck und vom Glanz des Himmels nichts stimmt. Ist das lyrisches Epigonentum? Jedenfalls hat der Dichter niemals erlebt, was er schildert, aber weil er auf der anderen Seite die eigene Erfahrung überhaupt nicht braucht, kann er alles schildern. Seine Arbeitsprinzipien sind Beliebigkeit und Variationslust, und das bringt mit sich, daß sehr vieles austauschbar ist. Denn eine innere Folgerichtigkeit ist nicht da und will auch nicht vorgetäuscht werden. Auch ganze Zyklen könnte man umstellen, ohne daß sich an der Aussage das geringste ändern würde. Nirgendwo ein Lebenskondensat, alles nur rhetorischlyrische Präsentation eines Schmerzes, den wir weder Rückert noch Heine abnehmen und von dem beide auch gar nicht glaubhaft machen wollen, dab er echt gewesen sei. Schmerz ist poetischer Rohstoff, und nur das. Diese Ausdünnung an realistischem Gehalt steht dabei in einer Wechselbeziehung zum erhöhten Pathos; Pathos ist der Versuch, durch Übertreibung sofort jeden Verdacht zu ersticken, daß hier etwas Wirkliches gemeint sein könnte. Pathos ist also nicht Ausdruck eines uneingestandenen Mangels, sondern ein Weg, um vom Erlebnis fortzukommen. Das Ende vom Lied ist ein Kunstbau, mit großer lyrischer Fertigkeit erstellt. Er macht dem Leser, der sucht, was nicht dahintersteht, schwer zu schaffen. Denn wer Individuelles finden will, stößt auf Allgemeinheiten und fühlt sich am Ende möglicherweise betrogen, hat er doch nur ein Sprechen in Klischees gehört, wo er vielleicht Unmittelbarkeit erwartete. Ein pausenloser lyrischer Theaterdonner geht bei Rückert auf den nieder, der vielleicht dankbar wäre für ein echtes Lebenszeichen. Aber das gibt es nicht in dieser Lyrik - man mul sie anders lesen. Bei Eichendorff fangt es schon an, daß einzelne Motive und typische Situationen dutzendfach, hundertfach durchgespielt werden, $d a ß$ in einem Waldgedicht allzu viele Register gezogen werden. Das Ende ist eine Alleskönnerschaft, für die Rückert wiederum das beste Beispiel liefert.

Rückert konnte wirklich alles. Nicht nur, daß er die klassischen indoeuropäischen Sprachen beherrschte, er wuBte sich in der Sprache der Tamilen und im Sanskrit zu Hause, hat das Drawidische studiert, persische und türkische Literatur. Das hat auch dem Lyriker Möglichkeiten eröffnet, um die ihn mancher beneidet haben dürfte. Haben wir uns einmal von der Vorstellung befreit, daß freie Verfügbarkeit Sprachverfall, daß Spiel mit Formeln Epigonentum sei, sind wir also aus dem Bannkreis des klassischen Normendenkens herausgeraten, so ist der ungeheure Reichtum an Formeln, Motiven, Situationen ein Glücksfall, kein poetisches Verhängnis. Rückert war ein lyrischer Akrobat hohen Grades, und er 
konnte in der Tat so ungefähr alles besingen, was ihm unter die Feder kam, und das auch in ungefähr allen Schattierungen und Aussageformen. Sein lyrisches Werk ist Alexandrinismus reinsten Wassers, aber man muB die ungeheure Bereicherung erkennen, die damit verbunden ist. Erlebnislyrik: eine ärmliche Selbstbeschäftigung, von hierher gesehen, klassische Normen und das "ächt Objektive" eine lyrische Schmalspurbahn, die in einer Sackgasse endet. Rückerts große Leistung ist die Amalgamation abendländischer und morgenländischer Kunstformen, von der Übertragung der östlichen Weltliteratur ins Deutsche ganz abgesehen. Man muB auch die ungeheure lyrische Kreativităt als Gewinn buchen, nicht als beliebige Fließbandproduktion von Gedichten. Nur eines darf man nicht dahinter suchen: erlebte Wirklichkeit. Wir könnten auch sagen: Mit Rückert hat die Lyrik wieder zu einem Reichtum zurückgefunden, der früher schon vorhanden gewesen, aber dann in der Klassik und bei den Klassizisten gründlich verlorengegangen war. Barocke Bilder, Anakreontik und Rokokolyrik, das Graziöse des 18. Jahrhunderts, Hexameter, Pentameter, Distichen, sapphische Oden, alkäische Oden, Sonette, Alexandriner - alles vorhanden. Rückerts Lyrik ist eine weltliterarische poetische Literaturgeschichte vom 17 . Jahrhundert bis zu seiner Gegenwart. Die lyrischen Traditionen wieder verfügbar gemacht zu haben, ist die große Leistung des 19. Jahrhunderts.

Am Ende dieser Entwicklung steht - vielleicht - Storm. Das Etikett, er sei ein Erlebnisdichter, überdies noch Husumer Prägung, ist eine für ihn tödliche Kategorie. Nur zu oft hat man bei Storm übersehen, welch außerordentlicher Reichtum an lyrischen Möglichkeiten auch in seiner Lyrik lebt. Das würde bei einem Erlebnisdichter ohnehin sonderbar anmuten. Auch Storm ist ein Virtuose auf der Klaviatur der lyrischen Überlieferungen; das romantische Gedicht und das Erlebnisgedicht sind nur zwei Möglichkeiten auf seinem poetischen Manual. Die Spruchdichtung Storms nutzt barocke Überlieferungen, ohne daß Storm dabei parodistisch geworden wäre, wie später Arno Holz das geliebt hat. Wir haben Gedichte ohne strophische Gliederung, Vierzeiler, Sonette, erzählende Gedichte, Ghaselen, Rätsel, Dialoggedichte, Chöre, Stammbuchblätter, Grabsprüche, Sinnsprüche in jeder Form, Gedichte in Märchenform oder vielmehr Märchen in Gedichtform; lyrische Zwiegesänge, Zweizeiler, Anakreontisches, Wortspiele, auch witzige Poesie im Sinne der Anakreontik. Mit den Erlebnisdichtern hat Storm nichts tun, aber ein lyrischer Verwandter Rückerts ist er ohne jede Frage. Leider hat selbst die Storm-Forschung ihn gelegentlich auf das „Konzept des Erlebnisgedichts" hin festgelegt - so konnte man vor einiger Zeit noch lesen. ${ }^{35}$

35 Vinçon 1972, S. 46. 
Schlimmes Mißverständnis, ein krasses Übersehen der chamäleonhaften Fähigkeiten und damit seiner lyrischen Produktivität. Dabei liegen die Konstruktionspläne der Gedichte offen zutage, das lyrische Geheimnis ist - zum Glück - herauseskamottiert. Wozu es also suchen?

Realitätssuche und Realitätsverweigerung - das ist die Spannweite einer Lyrik, die normalerweise als Epiphänomen des Sprachverfalls gilt. Das Ende eines Normensystems ist Paradoxalität. Konträre Tendenzen prägen das Bild der Lyrik im 19. Jahrhundert aber auch noch in anderer Hinsicht. Daß die Sprache im frühen 19. Jahrhundert gleichsam auszulaugen droht, ist verständlich. Das Ende von Sprachnorm führt nahezu zwangsläufig zu Verflachungsprozessen, zur Einebnung von Bedeutungen, zur Einengung von Aussagemöglichkeiten. Alles in allem: das Münzgeld der Worte wird abgegriffener. Das war für Rückert Voraussetzung seiner Sprachspiele, für den Heine der Nordseebilder Anlaß zu Neuprägungen. Beides sind Reaktionen auf die neuen sprachlichen Verfügbarkeiten, auf das Abgegriffensein des poetischen Münzgeldes.

Wenn nach 1800 auf der einen Seite also so etwas wie eine sprachliche Inflation einsetzt, die lyrische Sprache, mit Eichendorff beginnend, bedeutungsentleert erscheint, wann immer von Waldeinsamkeit, dem Rauschen der Bächlein, von Sehnsucht und dem Morgen die Rede ist (lauter Gedichtüberschriften Eichendorffs), so kommt im frühen 19. Jahrhundert - und auch das ist ein Vorteil des Sprachverfalls - ein gleichsam allegorisches Sprechen auf. Das mag teilweise auf die Zensur zurückgeführt werden: Eine doppelte Aussageform, eine aus Schutzgründen verdeckte Rede empfahl sich vor allem im politischen Bereich. Aber wir haben eine Allegorisierung der Sprache auch anderswo. Eichendorff macht hier wieder den Anfang. Wenn in "Der alte Garten” von dem Springbrunnen die Rede ist, der immerfort noch von der alten schönen Zeit plaudert, dann wissen wir, daß damit nicht nur die verlorene Kindheit gemeint ist, sondern mit dem Kindheitsgarten auch der verlorene Garten Eden. Das so unpräzise Bild von der im Springbrunnen symbolisierten „alten schönen Zeit" bekommt seinen Doppelsinn, oder besser: hinter der vordergründigen Bedeutung tut sich eine eigentliche auf. Wenn in ${ }_{n}$ Frische Fahrt" vom magisch wilden Fluß gesprochen wird und der Sänger von dem Glanze selig blind auf dem Strome fahren will, dann mag das die Donau oder auch der Rhein sein, auf jeden Fall ist es der Lebensstrom. Die Dämmerung bedeutet Zeitenwende, das Schiff ist ein Lebensschiff, hinter Rom erscheint Jerusalem. Mörike hat versucht, im Bild des Feuerreiters die Französische Revolution zu beschreiben, Eichendorff das gleiche Phänomen mit Hilfe einer Gewittermetaphorik, Kleist mit Hilfe des Bildes vom Erdbeben. Die Metaphorisierungskraft der Sprache ist 
in den ersten Jahrzehnten des 19. Jahrhunderts außerordentlich groß, Realien werden überall als Gleichnisse genutzt: der gallische Hahn auf dem Dache, rote Mützchen, der Blocksbergritt, ein dreifarbiges Zelt, das wilde Tier in der eigenen Brust. Der zeitgenössische Leser wuBte das sehr wohl in seiner doppelsinnigen Bedeutung zu erkennen.

Das alles war nichts Neues, gewissermaßen nur angewandte oder reproduzierte Aufklärung, die das allegorische Sprechen liebte. Winckelmann hatte schon seine Leser darüber belehrt, daß eine Allegorie aus sich selbst verständlich sei. Nun darf man im allegorischen Sprachgebrauch des frühen 19. Jahrhunderts aber nicht nur eine Nachwirkung der Aufklärung sehen. Im Doppelsinn einer Sprache steckt vielmehr ebenfalls Spielmaterial, das von den Schriftstellern gerne genutzt wurde. Auch darin zeigt sich die reproduktive Kraft der Sprache. Sprachinfiationäre Tendenzen also einerseits, der große Ausverkauf der Worte - auf der anderen Seite die Unterschichtung oberflächlicher Wortbedeutungen. Auch das ist zweifellos ein paradoxaler Prozeß. Er sollte hier, da er nicht ausführlich dargestellt werden kann, wenigstens umrissen sein.

Wo führt das alles hin? In die intellektuelle Poesie der Moderne, aber auch dahin, daß die Literatur jetzt für die Realität selbst genommen wird. Im 19. Jahrhundert sind schon die lyrischen Verfahren entwickelt, die wir später etwa bei T.S. Eliot in seinen ${ }_{n}$ Four Quartetts" oder im "Waste Land" finden, bei Rilke und bei George. Georges Zeile Kein ding sei wo das wort gebricht steht nicht nur am Ende der Erlebnisdichtung, sondern auch am Beginn der Moderne, die das Erlebnis nicht mehr braucht, um schreiben zu können. Wirklichkeit existiert nur in der Sprache, und es sind die angeblichen Epigonen, die Dilettanten, die Alexandrinisten vom Schlage Rückerts, die dem vorgearbeitet haben. Daß die Realität in den Worten sei, hat Sartre noch 1946 in seinem Essay über das Wesen der Literatur festgestellt. Hofmannsthals berühmter Chandos-Brief gehört ebenfalls in diesen Zusammenhang. Da wird auf höchst beredte Weise über die Grenze des Redens gesprochen, da wird in eleganter Sprache gesagt, daß die Sprache versage. Das ist richtig und falsch zugleich. Denn die Sprache versagt häufig, wo sie Wirklichkeit nachschreiben will, aber sie versagt nie, wo sie selbst als Wirklichkeit genommen wird. Das ist vielleicht der größte Gewinn des Sprachverfalls.

\section{Literat ur}

Brentano (1968): Clemens Brentano. Werke. 1. Bd. Hrsg. von Wolfgang Frühwald, Berhard Gajek, Friedhelm Kemp. München. 
Eichendorf (1957): Joseph Freiherr von Eichendorff. Gedichte. Epen. Dramen. Hrsg. von Gerhart Baumann in Verbindung mit Siegfried Grosse. Stuttgart.

Gutzkow (o.J.): Karl Gutzkow. Gesammelte Werke. 2. Ausgabe. 12 Bde. Jena.

Hauff (1970): Wilhelm Hauff. Sämtliche Werke in 3 Bänden. Nach den Originaldrucken und Handschriften. Bd. 3. München.

Heine (o.J.): Heinrich Heines Sämtliche Werke. Bd. 3. Hrsg. von Ernst Elster. Leipzig und Wien.

Heine (1975): Heinrich Heine. Historisch-Kritische Gesamtausgabe der Werke. Bd. I/1. Buch der Lieder. Bearb. von Pierre Grappin. Hamburg.

Heine (1978): Heinrich Heine. Historisch-Kritische Gesamtausgabe der Werke. Bd. 11. Ludwig Börne, Eine Denkschrift und Kleinere politische Schriften. Bearb. von Helmut Koopmann. Hamburg.

Immermann (1971): Karl Immermann. Werke in fünf Bänden. 1. Bd. Unter Mitarbeit von Hans Asbeck, Helga-Maleen Gerresheim, Helmut J. Schneider, Hartmut Steinecke hrsg. von Benno von Wiese. Frankfurt/M.

Lenau (1971): Nicolaus Lenau. Sämtliche Werke und Briefe. In zwei Bänden. 1. Bd. Gedichte und Versepen. Hrsg. von Walter Dietze. Frankfurt/M.

Rückert (1868): Friedrich Rückert's gesammeite poetische Werke in 12 Bänden. Bd. 1. Frankfurt/M.

Schiller (1958): Schillers Werke. Nationalausgabe. Bd. 22. Vermischte Schriften. Hrsg. von Herbert Meyer. Weimar.

Schiller (1963): Schillers Werke. Nationalausgabe. Bd. 21. Philosophische Schriften. 2. Teil. Unter Mitwirkung von Helmut Koopmann hrsg. von Benno von Wiese. Weimar.

Wellek/Warren (1956): Wellek, René/Warren, Austin: The Theory of Literature. 2. Aufl. (1. Aufl. 1942). New York.

Vinçon (1972): Vinçon, Hartmut: Theodor Storm in Selbstzeugnissen und Dokumenten. Hamburg. 